\title{
Centrifuge modelling of retaining walls embedded in saturated sand under seismic actions
}

\author{
Stefano Aversa, Luca de Sanctis, Rosa Maria Stefania Maiorano, \\ Michele Tricarico \\ Università degli Studi di Napoli "Parthenope" \\ Giulia Viggiani \\ Università degli Studi di Roma Tor Vergata \\ viggiani@uniroma2.it \\ Riccardo Conti \\ SISSA, International School for Advanced Studies \\ Gopal Madabhushi \\ University of Cambridge
}

\begin{abstract}
This paper describes the experimental work carried out under 'PROPWALL', a research project on the seismic performance of embedded walls in saturated sand carried out by Transnational Access to the Turner B eam Centrifuge of the University of Cambridge. The experimental equipment, the model preparation and the monitoring devices are described in detail. The results are presented in terms of accelerations, wall deflections, bending moments and excess pore pressures, as monitored by means of the devices installed on the walls and within the soil mass.
\end{abstract}

Acknowledgments The Authors are indebted to SERIES for granting access to the Turner B eam Centrifuge of CUED. The technical support of the research group at the Schofield Centre and in particular that of Mark Stringer and Charles Heron is also gratefully acknowledged.

\section{Introduction}

A number of cases are reported in the literature of damage or failure of gravity and embedded retaining walls during earthquakes, most of which occurred in saturated soils (Iai \& Kameoka, 1993; K amon et al., 1996; Fang et al., 2003; K oseki et al., 2012). As an example, Madabhushi \& Zeng (2007) reported the structural yielding of a cantilevered quay wall during the B huj earthquake of 2001, where significant ground settlements occurred in the backfill concurrently with a large outward movements of the wall. As observed by Zeng \& Steedman (1993), failure of retaining and quay walls during earthquakes, with associated damage to structures founded on the backfill, is caused either by a local failure in the structural members or by large displacements of the ground exceeding the serviceability conditions. It follows that two aspects are of major concern when dealing with the dy- 
namic behaviour of retaining walls, i.e., (i) the increase of internal forces in the wall due to the inertia forces acting into the soil, and (ii) the possible occurrence of permanent displacements due to the full mobilisation of the soil resistance.

Following the works by Okabe (1924) and Mononobe \& Matsuo (1929), several studies have been devoted to the problem of computing dynamic earth pressures on retaining structures, both with theoretical (Steedman \& Zeng, 1990; Lancellotta, 2007; Mylonakis, 2007; Kim et al., 2010) and experimental (A tik and Sitar, 2010) approaches. More recent work has focused on the dynamic behaviour of flexible embedded retaining walls (Madabhushi \& Zeng, 2007; Cilingir et al., 2011; Conti et al., 2012), and some of the major findings have been embodied in a more rational design of this type of structures in seismic conditions (Conti \& Viggiani, 2013). Relatively few experimental works, however, have considered the presence of water in the backfill (Zeng \& Steedman, 1993; Dewoolkar et al., 2001; Lee et al., 2005; Towhata et al., 2009), and most of them were devoted to the seismic behaviour of caisson and gravity retaining walls.

It is recognised by many authors that the presence of water in the soil can affect the seismic behaviour of retaining walls in three ways, that is (K ramer, 1996): (i) by altering the inertia forces within the backfill, (ii) by developing hydrodynamic pressures and (iii) by generating excess pore water pressures due to cyclic loading. To our knowledge, however, rational procedures to take into account these effects on the seismic design of retaining structures are still lacking, and the current practice usually relies on approximate, and quite crude, solutions (Matsuzawa et al., 1985).

This work presents the results of four centrifuge tests carried out in the geotechnical centrifuge of Cambridge University Engineering Department (CUED), under a TA (Transnational Access) proposal joining the two research groups of Università di Roma Tor Vergata and Napoli Parthenope (research project PROPWALL). The tests were performed on reduced scale models of pairs of retaining walls, both cantilevered and with one level of support near the top, embedded in saturated sand reconstituted at two different values of relative density. $B$ ased on a systematic comparison between experimental results obtained from models in dense sand and models in loose sand, the main objective of this work is to shed some light on the main mechanisms affecting the dynamic behaviour of embedded retaining walls in the presence of pore water pressures. Emphasis is given on how, and to which extent, the occurrence of excess pore water pressures and/or liquefaction phenomena within the soil can affect the behaviour of these structures during earthquakes, both from a structural and a geotechnical point of view.

In the following, all physical quantities are given at prototype scale, with the only exception of the geometrical dimensions of the models. Moreover, accelerations are positive rightwards and the horizontal displacements of the walls are positive towards the excavation. 


\section{Experimental setup and model preparation}

The experimental work included a set of four tests on embedded walls in saturated sand, with piezometric head at dredge level (see Figure 1). Two tests were carried out on pairs of cantilevered walls (CWU1 and CWU2), and two tests on models of propped walls (PWU1 and PWU2). The dimensions of the geotechnical structures and the frequency content were chosen to be representative of real problems. A centrifugal acceleration equal to $40 \mathrm{~g}$ was selected. As detailed in Table 1, the total length of the model retaining walls was $L=200 \mathrm{~mm}$, corresponding to a total length at prototype scale of $8 \mathrm{~m}$. Excavation depths, $h$, of $90 \mathrm{~mm}$ (CWU) and 140 $\mathrm{mm}$ (PWU) were considered, corresponding to $3.6 \mathrm{~m}$ and $5.6 \mathrm{~m}$ at prototype scale, which are very common in engineering practice. In particular, the total length of the walls, the excavation depth and the embedment depth, $d$, are similar to those al ready adopted in past experimental work on retaining walls (Conti et al. 2012), in order to isolate the effects of the saturation condition on the dynamic behaviour of this type of structures.
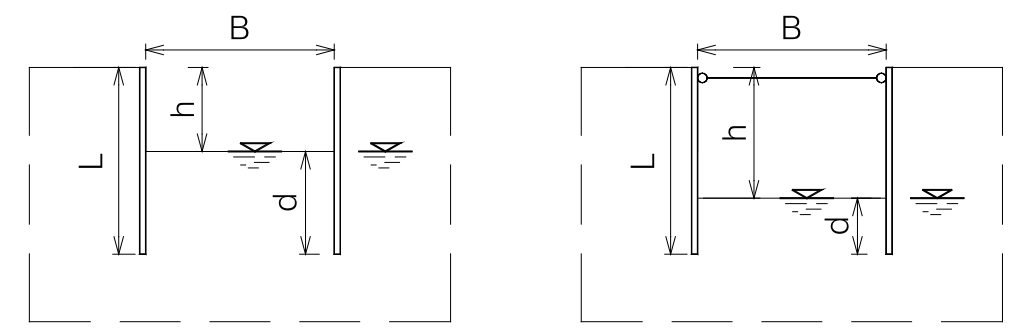

Fig. 1. Reference models and geometrical features: (a) tests CWU on cantilever ed walls and (b) tests PWU on propped walls

Table 1. Geometrical properties at model and prototype scale

\begin{tabular}{|c|c|c|c|c|c|c|c|c|c|c|c|c|}
\hline \multicolumn{4}{|c|}{} & \multicolumn{4}{|c|}{ Model scale (mm) } & \multicolumn{5}{|c|}{ Prototype scale (m) } \\
\hline Test & $\begin{array}{c}\mathrm{D}_{\mathrm{R}} \\
(\%)\end{array}$ & $N$ & $h$ & $d$ & $s$ & $Z$ & $B$ & $h$ & $d$ & $s$ & $Z$ & $B$ \\
\hline CWU1 & 38 & 40 & 90 & 110 & - & 400 & 200 & 3.6 & 4.4 & - & 10.8 & 8 \\
\hline CWU2 & 80 & 40 & 90 & 110 & - & 400 & 200 & 3.6 & 4.4 & - & 10.8 & 8 \\
\hline PWU1 & 38 & 40 & 140 & 60 & 9 & 400 & 200 & 5.6 & 2.4 & 0.45 & 10.8 & 8 \\
\hline PWU2 & 80 & 40 & 140 & 60 & 9 & 400 & 200 & 5.6 & 2.4 & 0.45 & 10.8 & 8 \\
\hline
\end{tabular}

\subsection{Seismic actuator and dynamic container}

Seismic excitations in the centrifuge tests are generated by a Stored Angular Momentum (SAM) actuator developed at Cambridge University (Madabhushi et al., 
1998). The SAM actuator can apply waves with frequencies in the range $30-50 \mathrm{~Hz}$. Therefore, at the centrifugal acceleration of $40 \mathrm{~g}$, the predominant frequency at prototype scale would be in the range $0.75-1.25 \mathrm{~Hz}$, which can be considered representative of real earthquakes, especially those characterized by a low frequency content.

To simulate boundary stress and deformation similar to those expected in a finite soil layer with indefinite lateral extent, the models were prepared in a Laminar Box (Knapett, 2006), consisting of the superposition of alternating aluminium frames and cylindrical bearings. The Laminar Box was designed in order to have negligible inertia, while being sufficiently stiff not to deform under high centrifugal accelerations (Brennan et al., 2006). The internal part of the laminar box is covered by a flexible rubber sheet to avoid loosing sand and/or pore fluid through the rings separating the frames.

\subsection{Materials and saturation procedure}

The model retaining walls consisted of aluminium alloy plates with thickness $t=6.36 \mathrm{~mm}$, corresponding to a bending stiffness at prototype scal e similar to that of a tangent concrete pile wall with a diameter of $400 \mathrm{~mm}$. For propped walls, two square aluminium rods with an axial stiffness of about $1 \times 10^{6} \mathrm{kN} / \mathrm{m}$ at prototype scale, connected to the walls by cylindrical hinges allowing rotation in the vertical plane, were located at a distance of $195 \mathrm{~mm}$ from each other.

A standard fine dry silica sand was used, namely Leighton Buzzard Sand $100 / 70$, fraction $\mathrm{E}$, reconstituted at two values of relative density, $D_{\mathrm{r}}=38 \%$ and $80 \%$. The specific gravity of the sand is $G s=2.65$, its maximum and minimum voids ratio are equal to 1.014 and 0.613 , respectively, while its constant volume friction angle is $\varphi_{c v}=32^{\circ}$ (Tan, 1990; Jeyatharan, 1991). Further details on the mechanical behaviour of the sand under monotonic, cyclic and dynamic loading conditions are given by Visone \& Santucci de Magistris (2009).

A high viscosity pore fluid was used, consisting of a methyl cellulose solution, to have a unique time scale for dynamic and diffusion phenomena. If the same pore fluid exists in the model and the prototype, the time scale for inertial effects and consolidation processes are $t_{\mathrm{m}}=t_{\mathrm{p}} / N$ and $t_{\mathrm{m}}=t_{\mathrm{p}} / N^{2}$ respectively. A possible strategy to solve this conflict is to scale the consolidation coefficient of the soil as $C_{l m}=C_{l p} / N$. Since

$c_{v}=\frac{k}{m_{v} \rho g}$ 
where $k$ is the permeability, $m$, the one-dimensional compressibility of the soil, $\rho$ the density of the pore fluid and $g$ the gravitational acceleration, a reduction in permeability will cause a direct reduction in $c_{v}$.

The permeability can be expressed as:

$k=\frac{K \rho g}{\mu}=\frac{K g}{v}$

where, $K$ is the intrinsic or absolute permeability of the soil, $\mu$ is the dynamic viscosity and $v=\mu / \rho$ is the kinematic viscosity. It is therefore possible to reduce the permeability of the soil $\mathrm{N}$ times by increasing the fluid viscosity by $\mathrm{N}$ times. To this end, in the past, silicone oil has been used as the pore fluid. However, this choice creates a number of experimental drawbacks, such as e.g., (i) silicone oil is classified as hazardous waste, and (ii) it is resistant to most solvents. The use of a solution of Hydroxyl-Propyl Methyl-Cellulose (HPMC) is an alternative choice (Stewart et al. 1998) that has a number of advantages: (i) methylcellulose is biodegradable; (ii) the components can be easily mixed with water to a wide range of viscosities; (iii) the unit weight of the solution is almost identical to that of pure water.

The models were saturated using an experimental set-up developed at Cambridge University (Stringer and Madabhushi, 2009). The CAM-sat (Fig. 2) is a computer-controlled system which is capable of: (i) monitoring continuously the rate of fluid entering the model; (ii) controlling the mass flow within specified targets; (iv) running for long periods without supervision.

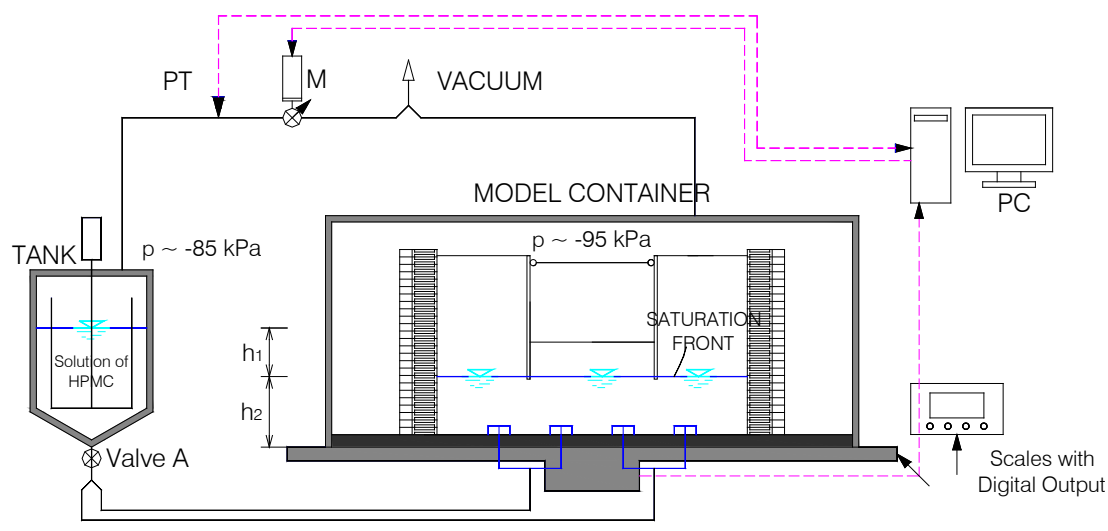

Fig. 2. CAM-sat system developed at Univer sity of Cambridge (after Stringer and Madabhushi 2009): PT (pressure transducer), M (motor).

A positive pressure difference drives fluid flow from a tank containing the viscous fluid into the model. The model is placed under vacuum to improve the de gree of saturation. The mass flow into the model is measured using a set of digital 
scales and the rate of saturation controlled by altering the vacuum applied to the reservoir.

\subsection{Instrumentation}

A fairly comprehensive instrumentation was mounted on the models to monitor the response of the soil-wall system during both the static and dynamic stages of the tests. In particular, accelerations at different locations within the soil mass and at the model boundaries were monitored using miniaturised piezoelectric accelerometers (Acc); pore water pressures at different locations around the excavation, both behind and between the walls, were measured using Pore Pressure Transducers (PPT); settlements of the soil surface behind the walls were measured using vertically mounted LVDTs. As far as the response of the structural elements is concerned, accelerations of the walls were monitored using Micro Electro $\mathrm{Me}$ chanical Systems (MEMS) accelerometers directly glued to the al uminium plates, bending moments were obtained from two sets of five strain gauges (SG) located al ong the central section of each wall, and, for propped walls, axial forces in the props were measured using two miniaturised load cells (LC) screwed in position in the central section of each prop. The horizontal displacements of each wall were measured using two LVDTs supported by a plate fixed to a gantry rigidly connected to the base of the box.

\section{Testing procedures}

At the beginning of the test, the centrifugal acceleration was increased in four successive steps of $10 \mathrm{~g}$ up to $40 \mathrm{~g}$. Once the testing acceleration of $40 \mathrm{~g}$ had been reached, the first earthquake was applied at the base of the model. During each test, the model was subjected to a series of trains of approximately sinusoidal waves with different nominal frequencies, $f$, amplitudes, $a_{\max }$, and durations, $t$. The basic idea was to apply earthquakes of increasing magnitude until the model walls suffered excessive rotation. Each earthquake was followed by a consolidation phase.

The first two tests were performed on cantilevered retaining walls. Test CWU1 was carried out on a loose sand layer $\left(D_{R}=38 \%\right)$, while test CWU2 was performed on dense sand $\left(D_{R}=80 \%\right)$. Figure 3 shows the cross section of models CWU1 and CWU2 and the layout of the instruments, which included 8 miniature piezoelectric accelerometers (Acc), 4 LVDTs for horizontal displacements, 2 LVDTs for vertical displacements, 8 (or 10) strain gauges for bending moments (SG), 4 (or 5) on each wall, and 8 pore pressure transducers (PPT). For test CWU2, instrumentation included also one potentiometer, to measure the settle- 
ment of the ground surface and one MEMS accelerometer, to measure the wall inclination at the end of both the static and the dynamic stages.

The acceleration recorded by accelerometer ACC 1 , placed on the lower frame of the Laminar Box, is considered as the input acceleration during the seismic stages.

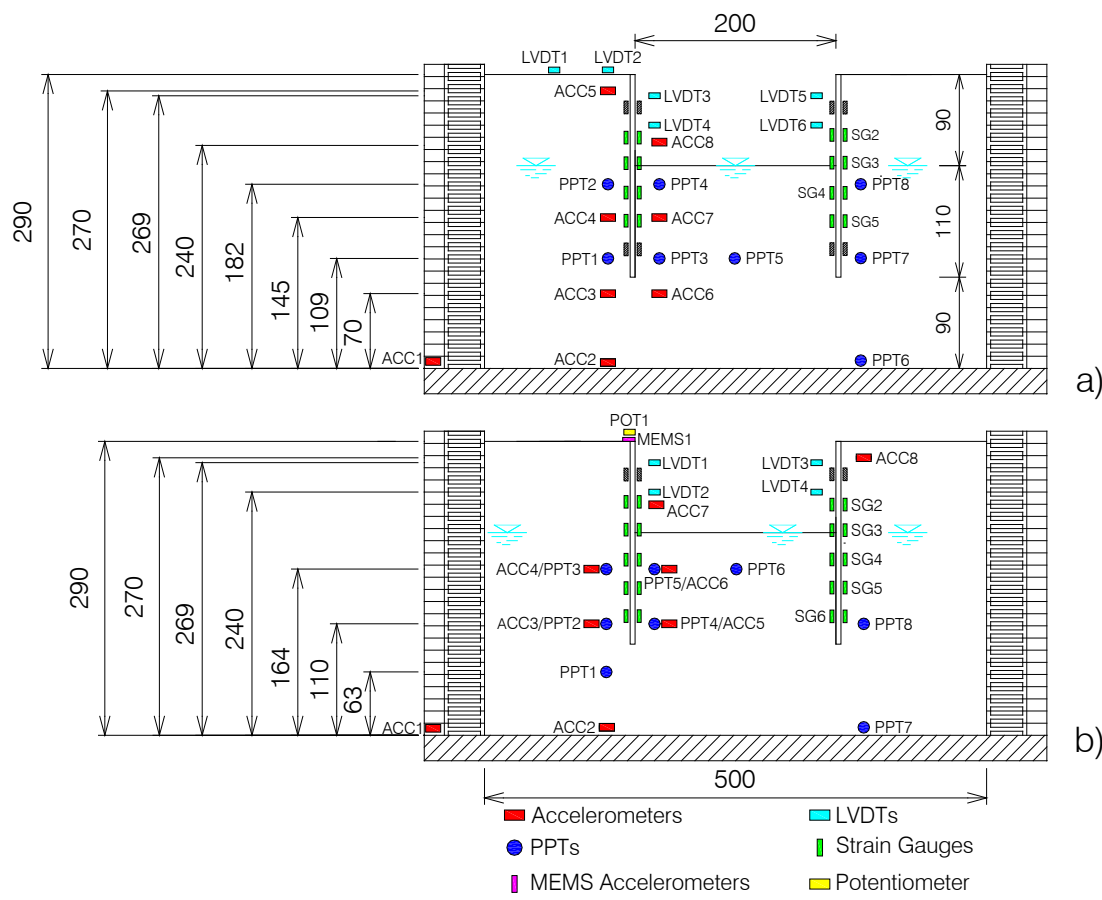

Fig. 3. Layout of instrumentation: (a) test CWU1 and (b) test CWU2.

In test CWU1 two earthquakes were applied (see Table 2 and Figure 4). The second earthquake was applied after two hours from the end of the first earthquake, to allow dissipation of excess pore pressures. In test CWU2, the model was excited by three earthquakes with gradual ly increasing amplitude, up to a value of $0.2 \mathrm{~g}$. The frequency of the waves was $50 \mathrm{~Hz}$ at model scale, i.e., $1.25 \mathrm{~Hz}$ at prototype scale. The duration of the signals was the same as that used for test CWU1 (Table 2 and Figure 4). 
Table2. Test sCWU1 and CWU2

\begin{tabular}{|l|l|l|l|l|}
\hline Test & Earthquake & $\mathrm{f}(\mathrm{Hz})$ & $\mathrm{a}_{\max }(\mathrm{g})$ & duration \\
\hline \multirow{3}{*}{ CWU1 } & EQ1 & 1.25 & 0.1 & 35 \\
\cline { 2 - 5 } & EQ2 & 1.25 & 0.2 & 35 \\
\hline \multirow{4}{*}{ CWU2 } & EQ1 & 1.25 & 0.075 & 33 \\
\cline { 2 - 5 } & EQ2 & 1.25 & 0.1 & 35 \\
\cline { 2 - 5 } & EQ3 & 1.25 & 0.2 & 35 \\
\hline
\end{tabular}

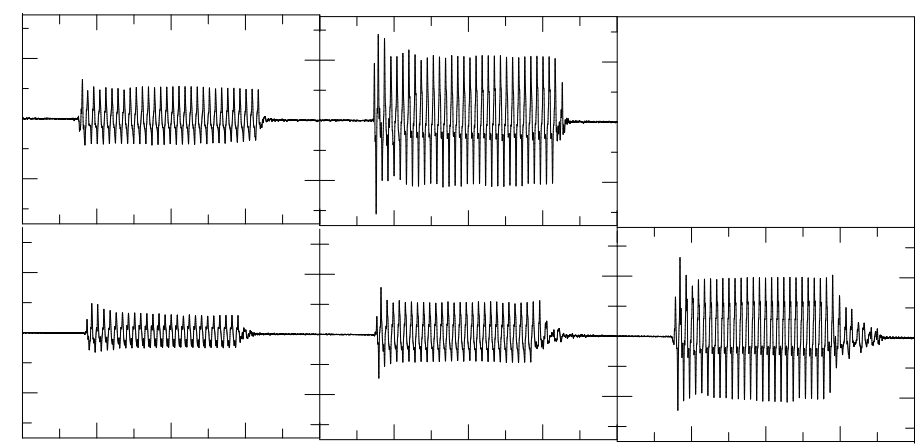

Fig. 4. Tests CWU1 and CWU2: input acceler ations.

The other two tests were performed on single propped retaining walls. Test PWU1 was carried out on loose sand $\left(D_{R}=38 \%\right)$, while test PWU2 was performed on dense sand $\left(D_{R}=80 \%\right)$. Figure 5 shows the cross section of tests PWU1 and PWU2 and the layout of the instruments, which included 8 miniature piezoelectric accelerometers (Acc), 4 LVDTS for horizontal displacements, 1 LVDT for vertical displacements, 10 strain gauges for bending moments, five on each wall, and 8 pore pressure transducers. Instrumentation included also 2 load cells to measure the axial force within the props.

Two earthquakes were applied in test PWU1 (Table 3), and three earthquakes with gradually increasing amplitude, up to a value of $0.2 \mathrm{~g}$ in test PWU2. The frequency of the waves was $50 \mathrm{~Hz}$ at model scale, i.e., $1.25 \mathrm{~Hz}$ at prototype scale. The duration of the signals is the same as that used in model test CWU1. 


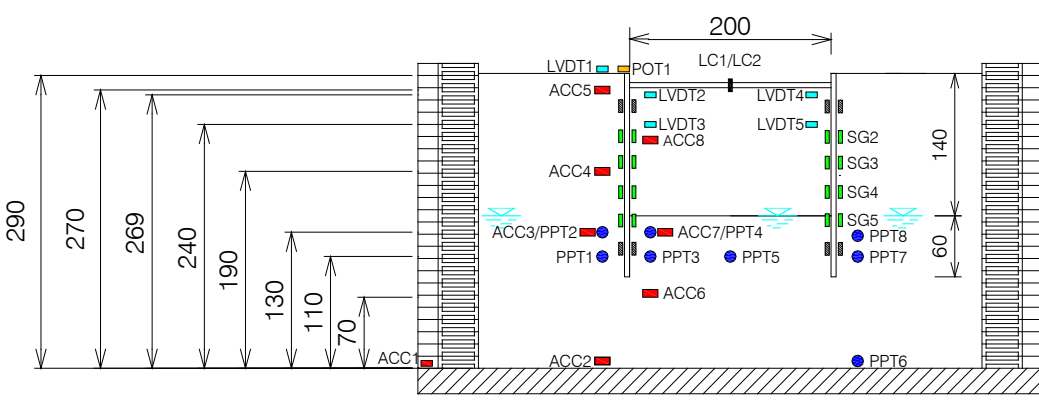

a)

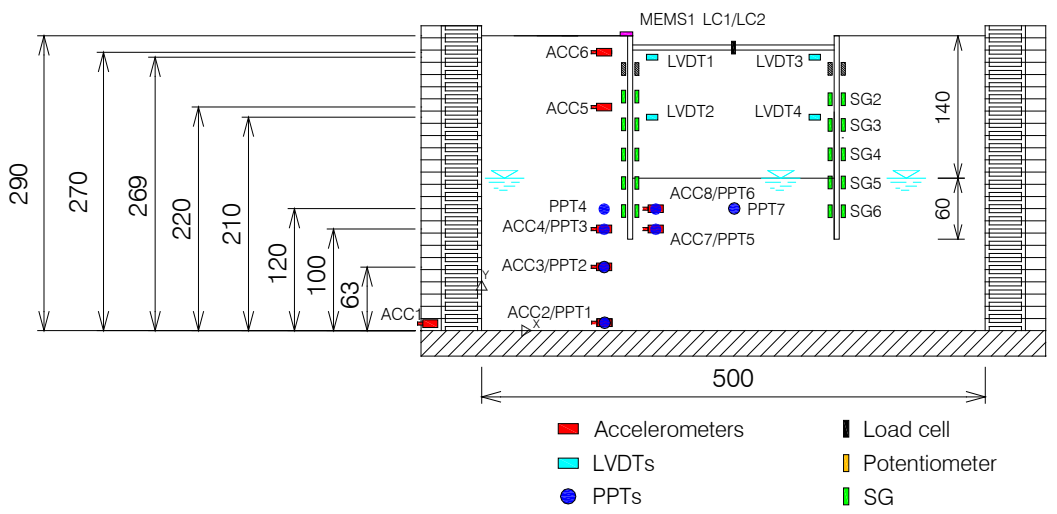

b)

Fig. 5. Layout of instrumentation: (a) test PWU1 and (b) test PWU2.

Table 3. Tests PWU1 and PWU2

\begin{tabular}{|l|l|l|l|l|}
\hline TEST & Earthquake & $\mathrm{f}(\mathrm{Hz})$ & $\mathrm{a}_{\max }(\mathrm{g})$ & duration \\
\hline \multirow{3}{*}{ PWU1 } & EQ1 & 1.25 & 0.1 & 35 \\
\cline { 2 - 5 } & EQ2 & 1.25 & 0.2 & 35 \\
\hline \multirow{3}{*}{ PWU2 } & EQ1 & 1.25 & 0.1 & 35 \\
\cline { 2 - 5 } & EQ2 & 1.25 & 0.13 & 35 \\
\cline { 2 - 5 } & EQ3 & 1.25 & 0.25 & 35 \\
\hline
\end{tabular}




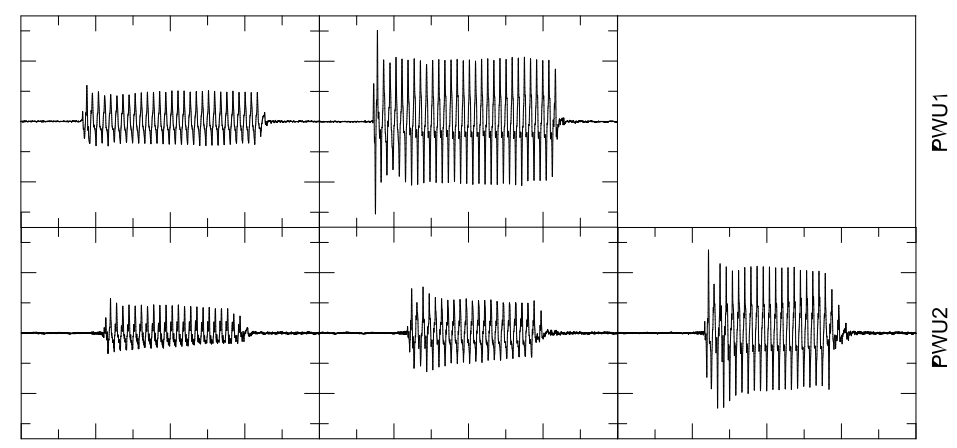

Fig.6 Tests PWU1 and PWU2 Input accelerations.

\section{Main results}

In this section the main experimental results are presented in terms of both accelerations and pore pressures within the soil layer, and displacements and internal forces in the structural members. Figure 7 shows a typical time history of a physical quantity (pore pressure, displacement, bending moment, axial force) measured during the applied earthquake, where the same terminology introduced by Dewoolkar et al. (2001) is adopted. The 'pre-seismic' value is the measurement at the beginning of the seismic event; the 'maximum' and 'minimum' are the maximum and minimum values that were reached during the test; the term 'dynamic' refers to the pre-seismic value and, concerning pore pressures, it coincides with 'excess'. The value measured immediately after shaking is defined as 'short-term residual' and, finally, the thick line toward the right side of the plot represents the 'longterm residual' value, recorded after the excess pore pressures are completely dissipated.

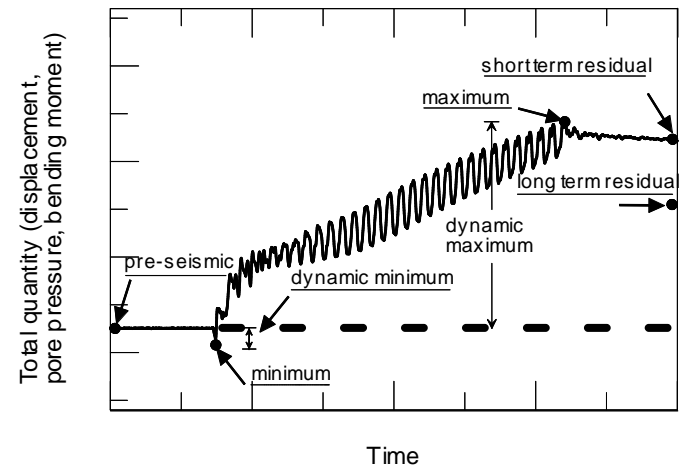

Fig. 7. Typical time history of a physical quantity measured during the applied earthquake and adopted terminology. 


\subsection{Accelerations}

Figure 8 shows the acceleration time histories measured at different depths within the model, both below and above the water table level, during tests (a) PWU1 (accelerometers Acc1, Acc4 and Acc5) and (b) PWU2 (accelerometers Acc1, Acc4 and Acc6). The input signals are clearly de-amplified when propagating through the saturated loose sand layer (Fig. 8(a)), the maximum accelerations recorded by Acc4 being about $0.04 \mathrm{~g}$ after the first cycles, irrespective of the amplitude of the applied earthquakes. On the other hand, the accelerations tend to be amplified again when travelling up to the soil surface (Acc5). A completely different scenario takes place during the three earthquakes of test PWU2 (Fig. 8(b)), where the input signals do not suffer marked de-amplifications through the saturated dense sand layer. These observations suggest that liquefaction phenomena occurred during test PWU1, leading to a dramatic reduction of the soil resistance and, consistently, to a systematic de-amplification of the horizontal accelerations.

The same behaviour was observed during the two tests on cantilevered walls. Figure 9 shows, for all the tests and the applied earthquakes, the maximum accelerations recorded close to the soil surface against the maximum value of the corre sponding input signals. The centrifuge data presented by Conti et al. (2010), obtained from a number of dynamic centrifuge tests carried out on dry sand, are also shown for comparison. Accelerations are always amplified when propagating through dry sand, irrespective of its initial density, while relative density clearly affects the response of the saturated soil models. In this case, in fact, a sort of cutoff is observed in the surface accelerations recorded in the loose models $\left(a_{\max } \approx 0.08 \mathrm{~g}\right)$, while substantial amplifications are still observed in the dense models.

\subsection{Pore pressures}

As shown in Figures 3 and 5, pore pressures were measured at different locations and depths within the soil layer. In order to check the reliability of PPT measurements, Figure 10 shows a comparison between the experimental values recorded at the end of the swing up stage, corresponding to the static pore pressure distribution, and those computed assuming an hydrostatic distribution of the fluid pressure. The agreement between measured and theoretical values is rather good, with an average scatter of about 19\%, probably due to the uncertainty in the exact location of the PPT transducers.

Figure 11 shows the time histories of the pore pressures measured during tests (a) PWU1 and (b) PWU2. Excess pore pressures developed during all the dynamic events, within both the dense and the loose model, with a faster rate in the first cycles and minor oscillations during the whole duration of the earthquake. After 
each shaking, the excess pore pressures dissipated and the long-term residual pore pressures were close to the corresponding static values.

A liquefaction indicator often adopted in the literature is the excess pore water pressure ratio, $r_{\mathrm{u}}=\Delta u / \sigma_{\mathrm{V}}^{\prime}$, defined as the ratio between the dynamic increment of pore water pressure and the vertical effective stress. For the centrifuge tests discussed in this paper, however, the complex stress distribution expected around the excavation makes a reliable estimation of this indicator difficult. Nevertheless, the occurrence of liquefaction phenomena in the loose sand layer (PWU1) was confirmed by a visual inspection of the model after the test, which revealed the presence of sand boils at dredge level between the walls (Fig. 12).
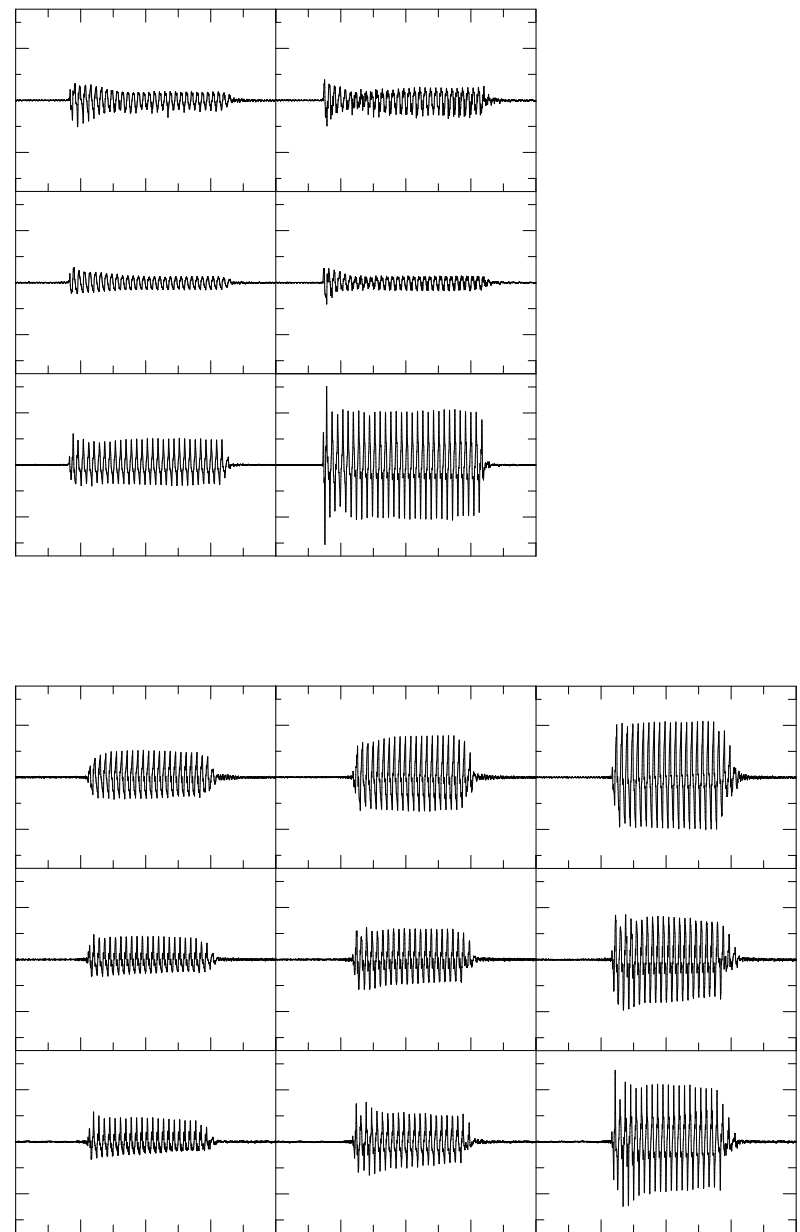

Fig. 8. Acceler ation time histories recor ded during tests: (a) PWU1 and (b) PWU2 


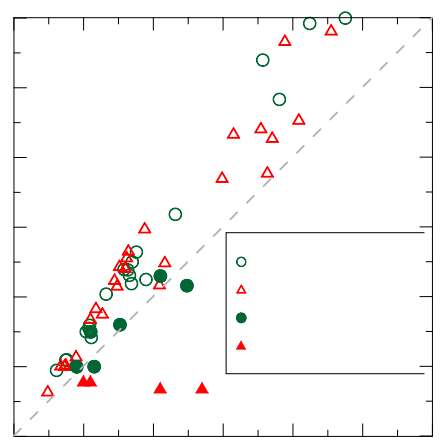

Fig. 9. Amplification of acceler ations: comparison between maximum acceler ations meas ur ed in dry sand models (Conti $e$ al., 2012) and those recor ded in the present tests

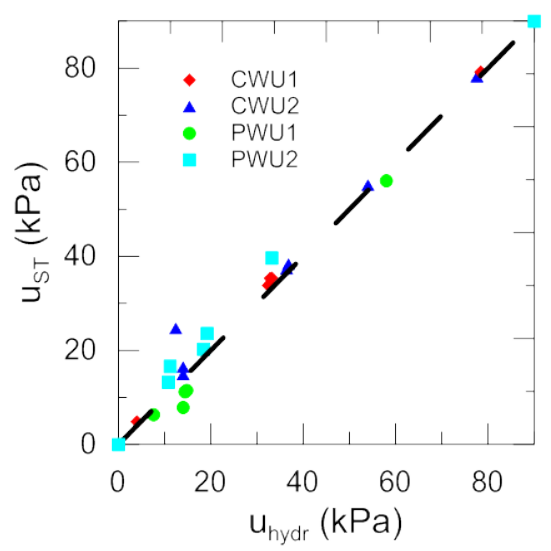

Fig. 10. Pore pr essures at the end of the swing up stage (static): comparison between theoretical values and experimental measur ements

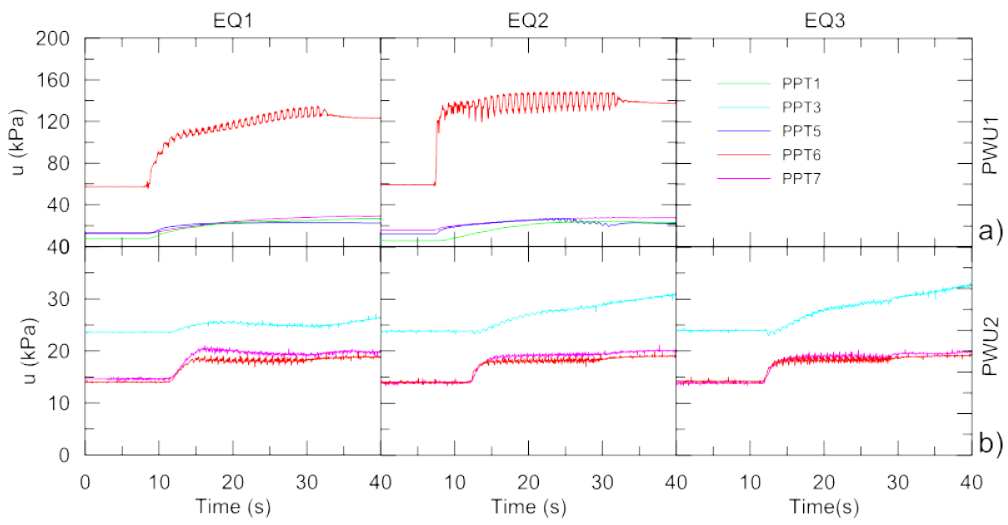

Fig. 11. Por e pressure time histories recor ded during tests: (a) PWU1 and (b) PWU2. 


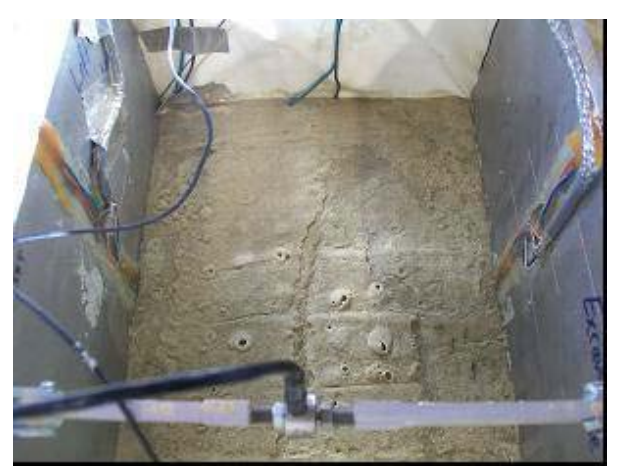

Fig. 12. Test PWU2. Photogr aph of the model, showing sand boils between the two walls, at dredge level.

\subsection{Displacements}

The interpretation of the behaviour of the embedded walls in terms of displacements is not immediate. If the walls are assumed to be rigid, a possible short-term residual deformed shape of the retaining walls relative to the base of the box (absolute displacements) is that depicted schematically in Figure 13(a). With respect to the configuration shown in Figure 13(a), the absolute displacements of the left and right wall, $\bar{u}_{L}$ and $\bar{u}_{k}$, measured by the LVDTs at any depth, can be expressed as follows:

$\bar{u}_{\mathrm{L}}=u_{\mathrm{L}}+u_{B \mathrm{~L}}=u_{\mathrm{L}}-u_{\mathrm{B}}$

$\bar{u}_{\mathrm{R}}=u_{\mathrm{R}}+u_{B \mathrm{R}}=u_{\mathrm{R}}+u_{\mathrm{B}}$

i.e. they are the sum of the displacement of the boundary of the laminar box, $u_{B}$, shown in Figure 13(b) and of the unknown displacements of the walls relative to their un-deformed configuration, $u_{L}$ and $u_{k}$, shown in Figure $13(d)$. Note that the displacements of the boundary of the box are shown as linear in Figure 13(b); this is not necessarily the case, but it is irrelevant in the interpretation of the data as the horizontal displacements of the wall are measured at only two positions and are interpreted as a rigid body motion. However, the boundary conditions imposed by the Laminar Box, similar to the condition of 'periodic' or 'tied-boundaries' (see Zienckiewicz et al. 1988), ensure that the displacements of the boundaries of the box at any depth, if non zero, are anti-symmetrical.

The relative displacement between the two walls can be computed from the displacements measured by the LVDTs as: 


$$
\Delta u=u_{\mathrm{R}}+u_{B \mathrm{R}}=\bar{u}_{\mathrm{R}}+\bar{u}_{\mathrm{B}}
$$

but, as the displacement of the boundary of the box is not measured during the test, it is not possible to work out the individual displacements of each wall relative to its undeformed configuration, unless some simplifying assumption is introduced. In this case, it was assumed that the displacements of the walls are 'symmetrical', i.e., $u_{k}=u_{\text {L }}$, see Figure 13(d). It follows that the displacements of each wall relative to the undeformed configuration can be obtained from the displacements measured by the LVDTs as:

$u_{\mathrm{L}}=u_{\mathrm{R}}=\frac{\bar{u}_{\mathrm{L}}+\bar{u}_{\mathrm{R}}}{2}$

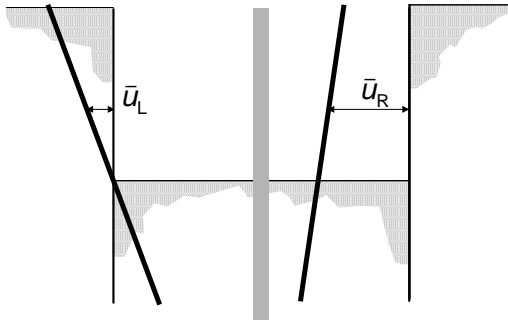

LVDT support

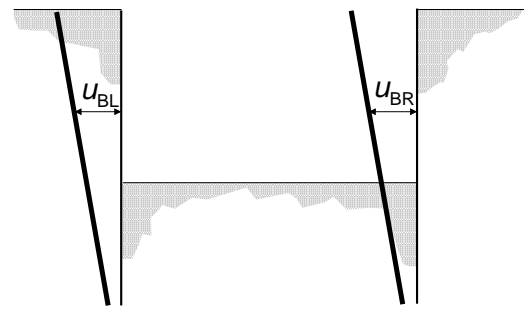

$u_{\mathrm{BR}}=-u_{\mathrm{BL}}=u_{\mathrm{B}}$

(b)
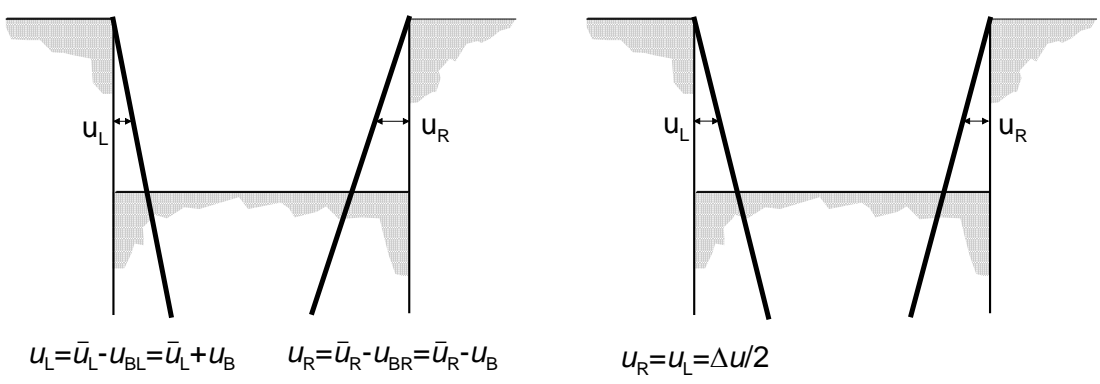

$u_{\mathrm{R}}=u_{\mathrm{L}}=\Delta u / 2$

(d)

Fig. 13. Inter pretation of LVDT measur ements of horizontal displacements of the walls. (a) absolute displacements, (b) assumed displacements of the box boundaries, (c) displacements of the walls relative to undefor med configur ation, (d) assumed symmetrical relative displacements.

This is quite a strong assumption, which may be not entirely realistic, particularly for non-symmetric acceleration time histories. However, the interpretation of wall displacements in terms of 'symmetrical' components is very convenient as it 
permits to emphasize the main mechanism of rotational failure of each wall under seismic actions.

The time-histories of the wall displacements measured by the LVDTs during tests PWU1 and PWU2 are plotted in Figure 14. In both tests: (i) the retaining walls accumulate permanent displacements; (ii) the short term- and long termresidual displacements are practically the same, with the exception of the left wall of test PWU1. The short- and long-term residual wall displacement profiles for PWU1 and PWU2 after the last earthquake (EQ2 for PWU1 and EQ3 for PWU2), as measured from the LVDTs, are plotted in Figure 15. For PWU1, the difference between short- and long-term displacements is worthy of note and is due to dissipation of earthquake-induced excess pore pressure. Symmetrical wall displacements are plotted in Figure 16. This representation permits a clearer perception of the failure mechanism induced by the earthquake, corresponding to an inward rotation of the toe of the wall.

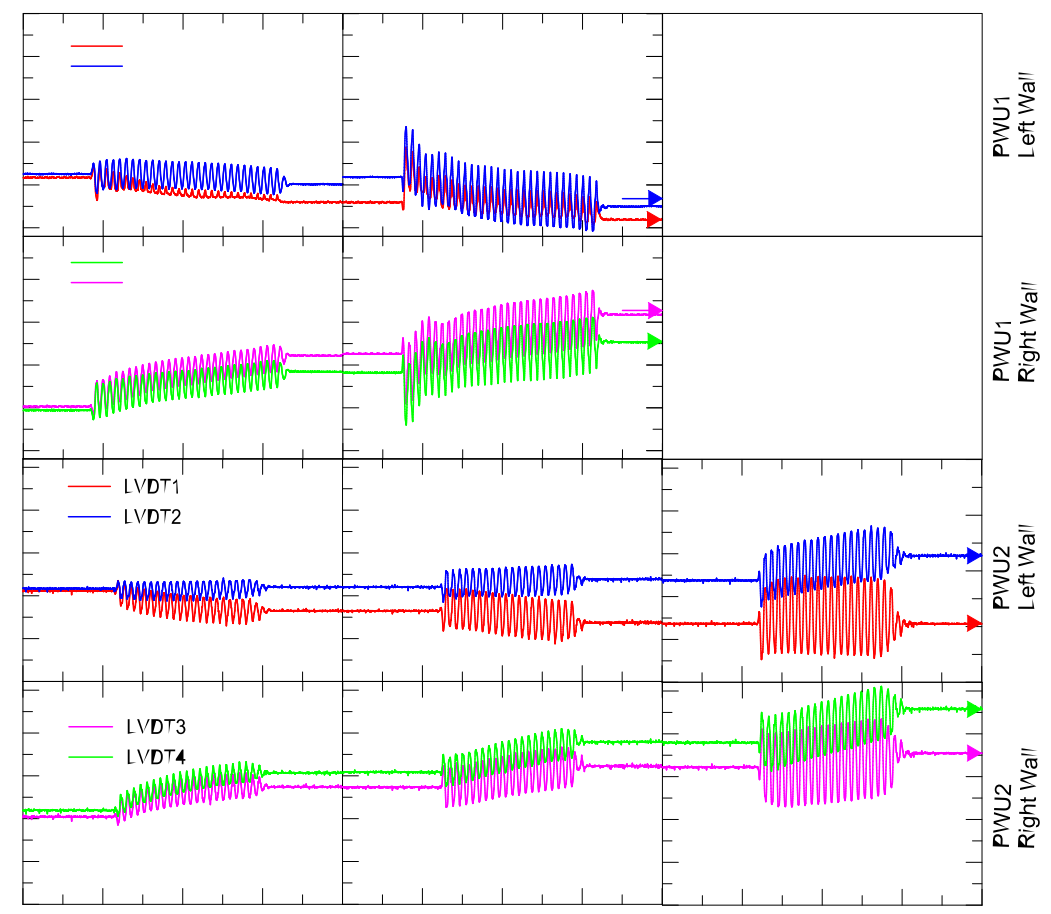

Fig. 14. Wall displacement time histories measured during tests: (a) PWU1 and (b) PWU2. 

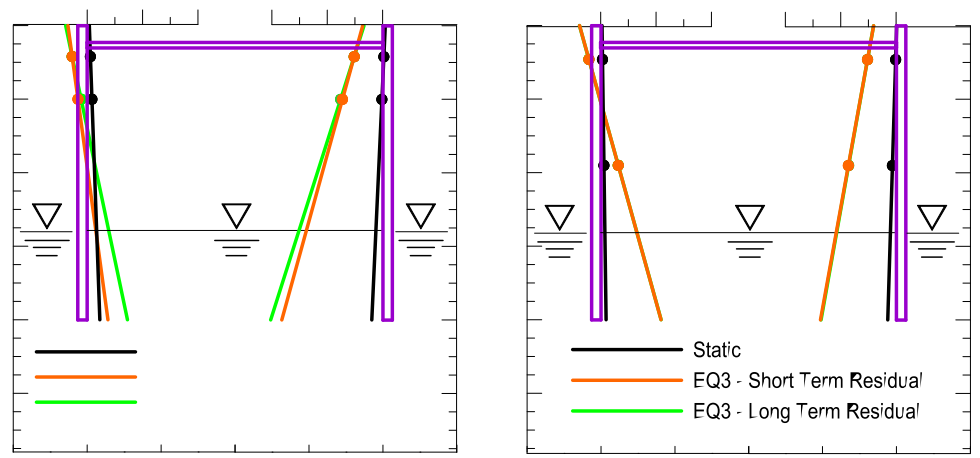

Fig. 15. Horizontal absolute wall displacements. Comparison between: (a) model tests PWU1 and (b) model test PWU2.
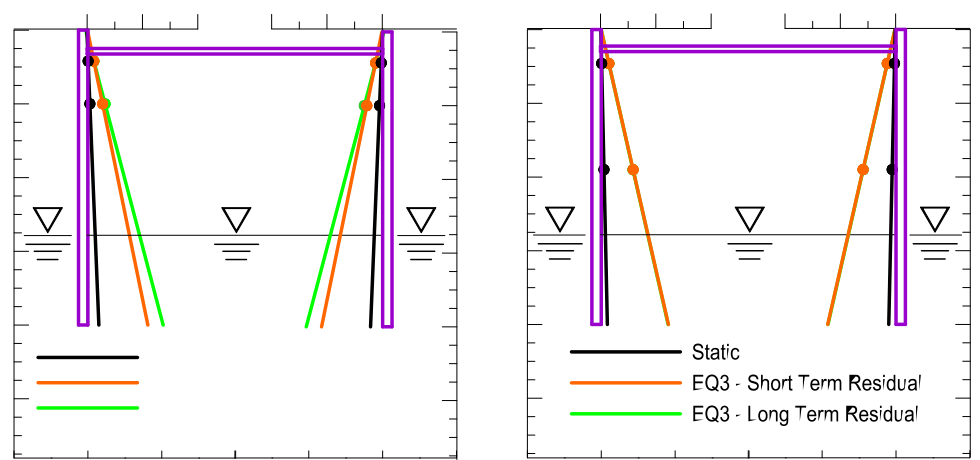

Fig. 16. Symmetrical displacements. Comparison between: (a) model test PWU1 and (b) model test PWU2.

\subsection{Bending moments}

Figures $17(\mathrm{a})$ and (b) show the time histories of bending moments measured on the right wall during tests PWU1 and PWU2, respectively. A completely different behaviour is observed between the two models. In test PWU2, the internal forces 
oscillate around an average value which continuously increases during shaking and the dynamic increments are proportional to the applied accelerations, consistently with other experimental work on embedded retaining walls in saturated sand (see e.g. Zeng \& Steedman, 1993; Madabhushi \& Zeng, 2007). On the other hand, during the transient stages of test PWU1, bending moments reduce substantially, reaching negative short-term residual values after EQ2. In both tests, dissipation of pore pressures after the earthquakes induces a redistribution of internal forces in the wall, the long-term residual values being, however, substantially different from the corresponding pre-earthquake values.

B ending moments on the walls clearly result from complex soil-structure interaction phenomena, in which many factors are likely to play a significant role, such as: the inertia forces into the soil, the excess pore pressure build-up and the consequent reduction in soil strength, the redistribution of stress and mobilisation of soil strength associated to wall displacements. While a full understanding of the problem based simply on centrifuge test results would be quite a hard task, the experimental observations suggest again that relative density affects significantly the dynamic response of retaining walls embedded in saturated sand.

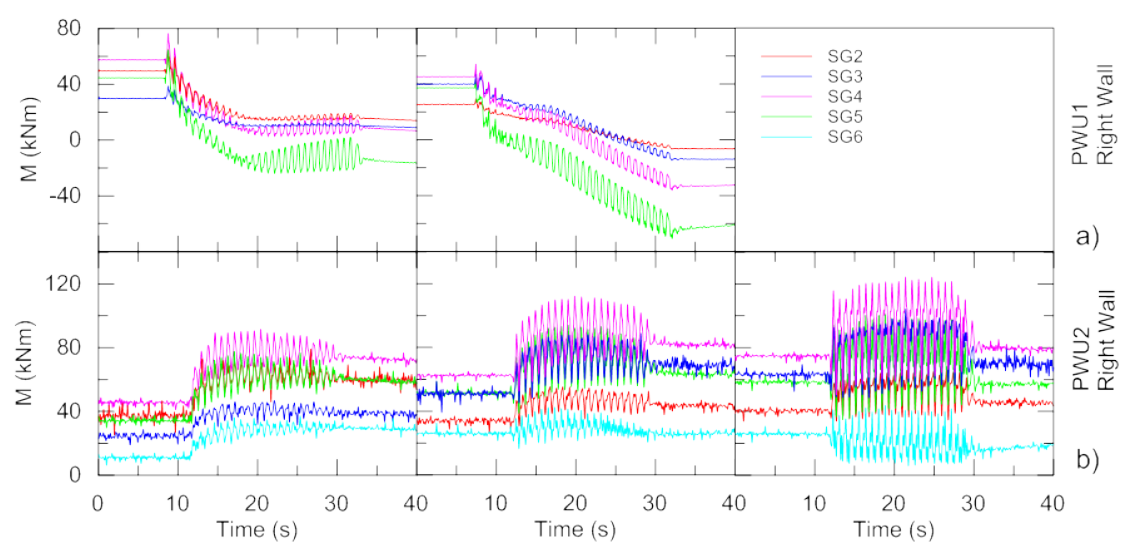

Fig. 17. Bending moments time histor ies measur ed on the right wall during tests: (a) PWU1 and (b) PWU2.

\section{Conclusions}

Four dynamic centrifuge tests were performed on pairs of retaining walls embedded in saturated sand, both cantilevered and with one level of support near the top. B oth the geometry of the system and the features of the applied earthquakes were similar to those considered in past experimental work on retaining walls embedded in dry sand, in order to emphasize the effects of saturation on the dynamic behaviour of such structures. 
The main results have been discussed in terms of accelerations and pore pressures into the soil, and internal forces and displacements of the walls. Indirect measurements and visual inspection of the models revealed that, while excess pore pressures developed in both dense and loose models, liquefaction phenomena occurred only in the latter case, leading temporarily to a dramatic reduction of soil resistance. This fact had two major effects, which make the overall behaviour of saturated loose models substantially different from that observed in dry and saturated dense models.

Firstly, the input signals were systematically de-amplified while propagating through the loose sand layers, with a sort of cut-off observed in the accelerations recorded close to the soil surface. On the contrary, the applied earthquakes were always amplified through the dense sand layers.

Secondly, inertia forces acting on saturated dense models induced dynamic increments of bending moments which are substantially proportional to the amplitude of the input accelerations. On the other hand, a significant reduction of internal forces was observed in the loose sand models, where even negative values of bending moments were reached during the transient stages.

It is evident that relative density affects significantly the seismic response of retaining walls embedded in saturated sand, where the excess pore pressures may lead to a substantial reduction of the soil resistance and, consequently, of the inertia forces into the soil. As a result, no increase of bending moments in the walls can be expected, as they strictly depend on both the inertia acting on the retaining side and on the soil passive resistance available below dredge level. From this perspective, the problem of computing the dynamic internal forces in the structural members become of little relevance, while the ability of predicting the permanent displacements experienced by the walls play a major role in the seismic design of such structures.

\section{References}

Atik L.A., Sitar N. (2010). "Seismic earth pressures on cantilever retaining structures". J. Geotech. Geoenv. Eng., 136(10), 1324-1333.

Brennan A.J., Madabhushi S.P.G., Houghton N.E. (2006). "Comparing Laminar and Equival ent Shear Beam (ESB) Containers for Dynamic Centrifuge Modeling". Proc. $6^{\text {th }}$ Int. Conf. Phys. Mod. Geotech., $6^{\text {th }}$ ICPMG ' 06, Hong Kong, 171-176.

Cilingir, U., Haigh, S.K., Madabhushi, S.P.G. and Zeng, X. (2011). "Seismic behaviour of anchored quay walls with dry backfill". Geomechanics and Geoengineering, 6(3), 227-235.

Conti R., Madabhushi S.P.G., Viggiani G.M.B., (2010). "Physical modelling of flexible retaining walls under seismic actions". Proc. $7^{\text {th }}$ Int. Conf. Phys. Mod. Geotech., $7^{\text {th }}$ ICPMG ' 10 , Zurich, Switzerland.

Conti R., Madabhushi S.P.G., Viggiani G.M.B. (2012). "On the behaviour of flexible retaining walls under seismic actions". Géotechnique, 62(12), 1081-1094.

Conti R., Viggiani G.M.B. (2013). "A new limit equilibrium method for the pseudostatic design of embedded cantilevered retaining walls". Soil Dyn. Earthquake Eng.. 50, 143-150. 
Dewoolkar M.M., Ko H.Y., Pak R.Y.S. (2001). "Seismic behaviour of cantilever retaining walls with liquefiable backfills." J. Geotech. Geoenviron. Eng., 127(5), 424-435.

Fang Y.S., Y ang Y.C., Chen T.J. (2003). "Retaining walls damaged in the Chi-Chi earthquake". Can. Geotech. J., 40, 1142-1153.

Iai, S. and Kameoka, T. (1993). "Finite element analysis of earthquake induced damage to anchored sheet pile quay walls". Soils Found., 33(1), 71-91.

Jeyatharan K. (1991). "Partial liquefaction of sand fill in a mobile arctic caisson under dynamic ice-loading". PhD thesis, University of Cambridge, UK.

Kamon M., Wako T., Isemura K., Sawa K., Mimura M., Tateyama K., Kobayashi S. (1996). "Geotechnical disasters on the waterfront". Soils Found., Special issue, January, 137-147.

Kim W.C., Park D., Kim B. (2010). "Development of a generalised formula for dynamic active earth pressure". Géotechnique, 60(9), 723-727.

K nappett J. A. (2006). Piled foundations in liquefiable soils: accounting for axial loads. PhD Thesis, Cambridge University, England.

Koseki J., Koda M., Matsuo S., Takasaki H., Fujiwara T., (2012), "Damage to railway earth structures and foundations caused by the 2011 off the Pacific Coast of Tohoku Earthquake". Soils and Found., 52(5), 872-889.

K ramer S.L. (1996). "Geotechnical earthquake engineering". Upper Saddle River, New Jersey, Prentice Hall.

Lancellotta R. (2007). "Lower-bound approach for seismic passive earth resistance". Géotechnique, 57(3), 319-321.

Lee C.J. (2005). "Centrifuge modeling of the behavior of caisson-type quay walls during earthquakes". Soil Dyn. Earthquake Eng.. 25, 117-131.

Madabhushi, S. P. G., Schofield, A. N. \& Lesley, S. (1998). "A new stored angular momentum (SAM) based earthquake actuator". Proc. Centrifuge '98, Int. Conf. on Centrifuge Modelling, Tokyo, 111-116.

Madabhushi S.P.G., Stringer M.E., (2009). "Novel Computer - Controlled Saturation of Dynamic Centrifuge Models Using High V iscosity Fluids, Geotechnical Testing Journal, V ol. $32, \mathrm{~N}^{\circ} 6$, GTJ102435.

Madabhushi, S.P.G., and Zeng, X. (2007). "Simulating Seismic Response of Cantilever Retaining Walls". J. Geotech. Geoenv. Eng., 133(5), 539-549.

Matsuzawa H., I shibashi I., Kawamura M. (1985). "Dynamic soil and water pressures of submerged soils". J. Geotech. Eng., ASCE, 111(10), 1161-1176.

Mylonakis, G., Kloukinas, P., Papantonopoulos, C. (2007). "An alternative to the MononobeOkabe equations for seismic earth pressures". Soil Dynamics and Earthquake Engineering 27 (10), pp. 957-969

Mononobe N., Matsuo H. (1929). "On the determination of earth pressure during earthquake". Proc. 2nd W orld Engineering Conference, V ol. 9, 177-185.

Okabe, S. (1924). "General theory of earth pressure and seismic stability of retaining wall and dam". Journal of Japanese Society of Civil Engineering, 12(1).

Steedman R.S., Zeng X. (1990). "The influence of phase on the calculation of pseudo-static earth pressure on retaining wall". Géotechnique, 40(1), 103-112.

Stewart, D. P., Chen, Y. R., and K utter, B. L. (1998), "Experience with the Use of Methylcellulose as a Viscous Pore Fluid in Centrifuge Models". Geotechnical Testing Journal, 21(4), 365-369.

Stringer M.E., and Madabhushi S.P-G. (2009). "Novel Computer-Controlled Saturation of Dynamic Centrifuge Models Using High V iscosity Fluids". Geotechnical Testing Journal, Vol. 32(6).

Takahashi, H., K itazume, M., I shibashi, S., and Y amawaki, S., (2006). "Evaluating the Saturation of Model Ground by P-Wave Velocity and Modelling of Models for a Liquefaction Study," Int. J. Phys. Modell. Geotech., Vol. 6, No. 1, pp. 13-25.

Tan F. S. C. (1990). Centrifuge and theoretical modelling of conical footings on sand. PhD Thesis, Cambridge University, England. 
Towhata I., Alam M.J., Honda T., Tamate S. (2009). "Model tests on behaviour of gravity-type quay walls subjected to strong shaking". Bull. New Zealand Soc. Earthquake. Eng., 42(1), 47-56.

Visone, C., Santucci de Magistris, F. (2009). "Mechanical behaviour of the Leighton B uzzard Sand 100/170 under monotonic, cyclic and dynamic loading conditions". ANIDIS, B ologna.

Zeng, X., Steedman, R.S. (1993). "On the behaviour of quay walls in earthquakes". Géotechnique, 43(3), 417-431.

Zienckiewicz, O.C., Bicanic, N., and Shen F.Q. (1988). "Earthquake input definition and the transmitting boundary conditions". Conference on Advances in Computational Non-Linear Mechanics: 109-130. Editor St. Doltnis I, 1988. 